TRANSACTIONS OF THE

AMERICAN MATHEMATICAL SOCIETY

Volume 327, Number 1, September 1991

\title{
FLOWBOX MANIFOLDS
}

\author{
J. M. AARTS AND L. G. OVERSTEEGEN
}

\begin{abstract}
A separable and metrizable space $X$ is called a flowbox manifold if there exists a base for the open sets each of whose elements has a product structure with the reals $\Re$ as a factor such that a natural consistency condition is met. We show how flowbox manifolds can be divided into orientable and nonorientable ones. We prove that a space $X$ is an orientable flowbox manifold if and only if $X$ can be endowed with the structure of a flow without restpoints. In this way we generalize Whitney's theory of regular families of curves so as to include self-entwined curves in general separable metric spaces.

All spaces under consideration are separable and metrizable.
\end{abstract}

\section{INTRODUCTION}

0.1 . Whitney's paper Regular families of curves [W] has been of great influence. It laid the groundwork for the theory of cross-sections in flows and it can also be seen as a prelude to the theory of foliations. In Whitney's terminology a curve is a topological copy of an interval (open, closed, or half-open) or a circle. A family of curves is a partition of a separable metric space into curves. The family is called regular if for every $\varepsilon>0$ and every point $p$ there is a $\delta>0$ such that, whenever $d(p, q)<\delta$, arbitrarily long arcs that are contained in the curve through $p$ can be pushed onto a similar arc through $q$ by a homeomorphism that moves the points of the arc not more than $\varepsilon$. Using these two properties Whitney proved the existence of cross-sections. He also showed that if the family of curves is orientable and if the space is locally compact, it is possible to endow the space with the structure of a flow in such a way that the open curves and the endpoints of curves coincide with the orbits of the flow.

0.2 . In this paper we present a generalization in two directions of these results. First, we allow the curves to be self-entwined and only require that there is a continuous bijection of $\Re$ (or $S^{1}$ ) onto the curve. Second, we perform the construction of a flow for any separable metric space. It seems that our proof has the additional benefit of being less complicated.

0.3. We found it more convenient to start our theory with a discussion of the local product structure instead of beginning with regular families of curves. This

Received by the editors February 1, 1990. The results of this paper were presented at the Joint Summer Research Conference in the Mathematical Sciences, Arcata, California, June 1989.

1980 Mathematics Subject Classification (1985 Revision). Primary 54H20, 54E99.

The second author was supported in part by NSF-DMS- 8602400 . 
is formalized in the definition of a flowbox manifold, a notion that encompasses the notions of dimension-one foliation and flow. In passing we observe that the importance of the local product structure has been noticed by various authors, e.g., [G, MR].

0.4. A flowbox manifold is a space in which every point has arbitrarily small neighborhoods with a product structure in such a way that a natural consistency condition is satisfied. The basic results about flowbox manifolds are collected in $\S 1$. In $\S 2$ we examine the dimension-one foliation of the flowbox manifold and introduce the notion of orientability. We carefully analyze the intersection of flowboxes in $\S 3$. Then we are in a position to prove our main result.

0.5. Main Theorem. Let $X$ be an orientable flowbox manifold. Then there is a flow on $X$ such that each streamline in $X$ is contained in some orbit of the flow.

The converse of the main theorem is well known (see Examples 1.4 and 2.12).

0.6 . The proof of the main theorem is carried out in $\S 4$. In $\S 5$ we extend Whitney's original definition of a regular family so as to include more general curves and spaces. The relation with work on matchbox manifolds is briefly discussed.

\section{FLOWBOX MANIFOLDS}

We discuss the basic properties of flowbox manifolds.

1.1. Definition. A separable metric space $X$ is called a flowbox manifold if it has the following two properties.

(1) Local product structure: there exists a base $\mathscr{U}=\left\{U_{\beta} \mid \beta \in B\right\}$ for the open subsets such that for each $\beta \in B$ there exists a space $S_{\beta}$ and a homeomorphism $h_{\beta}: S_{\beta} \times \Re \rightarrow U_{\beta}$.

(2) Consistency: suppose that $U_{\alpha}=h_{\alpha}\left(S_{\alpha} \times \Re\right)$ and $U_{\beta}=h_{\beta}\left(S_{\beta} \times \Re\right)$ are elements of $\mathscr{U}$. If $U_{\alpha} \subset U_{\beta}$, then for each $s \in S_{\alpha}$ there exists $t \in S_{\beta}$ such that $h_{\alpha}(\{s\} \times \Re) \subset h_{\beta}(\{t\} \times \Re)$.

We first present some examples of flowbox manifolds.

1.2. Matchbox manifolds. According to [AM] a space $X$ is called a matchbox manifold if for each $x \in X$ there is a zero-dimensional space $S_{x}$ such that $S_{x} \times \Re$ is homeomorphic to an open neighborhood of $x$. It follows that there are arbitrarily small such neighborhoods. It is to be noted that the components of $S_{x} \times \Re$ are precisely the sets $\{z\} \times \Re, z \in S_{x}$. From this it easily follows that condition (2) is satisfied. Consequently each matchbox manifold is a flowbox manifold.

1.3. Foliations. A dimension-one $C^{0}$-foliation of an $n$-dimensional manifold $M$ may be defined as a partition $\mathscr{L}=\left\{L_{\alpha} \mid \alpha \in A\right\}$ of $M$ into one-dimensional 
sets (the leaves of the foliation) such that for each point $x \in M$ there is a homeomorphism (a chart) $h_{x}: U \rightarrow \Re^{n-1} \times \Re$ which takes an open neighborhood $U$ of $x$ onto $\Re^{n}$ such that for each $\alpha$ each component of $L_{\alpha} \cap U$ is mapped onto $\{y\} \times \Re$ for some $y \in \Re^{n-1}$ and the family of all such charts forms an atlas for the manifold. It is easily seen that a dimension-one $C^{0}$-foliation is a flowbox manifold. See $[\mathrm{CN}]$ for more information about foliations.

1.4. Flows. Let $\pi: X \times \Re \rightarrow X$ be any flow (continuous dynamical system) without restpoints (see, e.g., $[\mathrm{H} 1, \mathrm{NS}])$. Then $X$ is a flowbox manifold. To show that this is correct we note that for each $x \in X$ there is a closed subset $B_{x}$ of $X$-called a local section at $x$-and a real number $\eta>0$ such that $x \in B_{x}$ and $\pi$ maps $B_{x} \times[-\eta, \eta]$ homeomorphically onto a neighborhood of $x$. See $[\mathrm{H} 1, \mathrm{AM}]$ for more details.

1.5. Standing notation. Let $S$ be any space. We define $F_{S}=S \times[-1,1]$ and $E_{S}=S \times(-1,1)$. The space $F_{S}$ is called a standard flowbox. For each $x \in S$ the set $\{x\} \times[-1,1]$ is called a streamline of $F_{S}$. The natural projections of $F_{S}$ onto $S$ and $[-1,1]$ are denoted by $\mathrm{pr}_{1}$ and $\mathrm{pr}_{2}$ respectively. Both $\mathrm{pr}_{1}$ and $\mathrm{pr}_{2}$ are open. As $[-1,1]$ is compact, $\mathrm{pr}_{1}$ is closed as well.

We now define flowboxes which play a very important role in the development of the theory.

1.6. Definitions. Let $U_{\beta}$ be an open set witnessing the local product structure of the flowbox manifold $X$, i.e., $U_{\beta}=h_{\beta}\left(S_{\beta} \times \Re\right)$ for some $\beta \in B$. A closed subset $V$ of $X$, which is contained in $U_{\beta}$, is called a flowbox if there exists a space $S$, a dense subspace $S^{0}$ of $S$, and a topological embedding $\phi: F_{S} \rightarrow X$ such that the following hold:

(1) $V=\phi\left(F_{S}\right)$ and $\operatorname{int}_{X} V=\phi\left(E_{S^{0}}\right)$;

(2) for each $y \in S$ there is a $z \in S_{\beta}$ with $\phi(\{y\} \times[-1,1]) \subset h_{\beta}(\{z\} \times \Re)$.

For each point $p \in \operatorname{int}_{X} V$ the set $V$ is also called a flowbox neighborhood of $p$. The induced map $\phi: F_{S} \rightarrow V$ is called a parameterization of $V$. The sets $\phi(\{y\} \times[-1,1]), y \in S$, are called streamlines of $V$ or of $X$.

It is to be noted that every flowbox $V$ of a flowbox manifold $X$ is a regular closed set, i.e., $V=\mathrm{cl}_{X}$ int $_{X} V$. We shall now discuss the existence of flowboxes.

1.7. Proposition. Suppose that $W$ is a neighborhood of $x$ in a flowbox manifold $X$. Then there is a flowbox neighborhood $V$ of $x$ such that $x \in V \subset W$. Proof. In accordance with (1) in Definition 1.1, let $U_{\beta}=h_{\beta}\left(S_{\beta} \times \Re\right)$ be an open set containing $x$. We may assume that $W$ is closed and $W \subset U_{\beta}$. Applying a translation parallel to $\Re$ we may also assume that $x=h_{\beta}(y, 0)$ for some $y \in S_{\beta}$. The set $h_{\beta}^{-1}(W)$ is a closed neighborhood of $(y, 0)$ in $S_{\beta} \times \Re$. Let $S^{0}$ be a regular open subset of $S_{\beta}$ such that $y \in S^{0}$ and $S^{0} \times(-\varepsilon, \varepsilon) \subset h_{\beta}^{-1}(W)$ for some $\varepsilon>0$. We write $S=\mathrm{cl}_{S_{\beta}}\left(S^{0}\right)$. Without difficulty one verifies that $\phi: F_{S} \rightarrow V$ defined by $\phi(z, t)=h_{\beta}(z, t \varepsilon)$ is a parameterization of $V$. 
As an immediate consequence we obtain the following result.

1.8. Proposition and Definition. Let $X$ be a flowbox manifold. Then there exists a countable collection $\mathscr{V}=\left\{V_{i} \mid i=1,2, \ldots\right\}$ of flowboxes $V_{i}=\phi_{i}\left(F_{S_{i}}\right)$ such that the collection $\left\{\phi_{i}\left(E_{S_{i}^{0}}\right) \mid i=1,2, \ldots\right\}$ is a base for the open sets. Such a base $\mathscr{V}$ is called a flowbox base of $X$.

The following propositions are obvious (cf. [AM, 1.4]).

1.9. Proposition. Suppose that $\left\{V_{\alpha} \mid \alpha \in A\right\}$ is a discrete collection of flowboxes in $X$. Then $\bigcup\left\{V_{\alpha} \mid \alpha \in A\right\}$ is a flowbox in $X$.

1.10. Proposition. An open subset of a flowbox manifold is a flowbox manifold.

\section{ORIENTATION}

In the process of defining a flow structure on a flowbox manifold we have to single out the sets which will become the orbits.

2.1. Definition. Let $J$ be an arc in a flowbox manifold $X$ and let $g:[0,1] \rightarrow$ $J$ be a topological embedding. Then $J$ is called a partial orbit of $X$ if for every $t \in(0,1)$ there exists a flowbox neighborhood $V=\phi\left(F_{S}\right)$ of $g(t)$ such that $V \cap J=\phi(\{x\} \times[-1,1])$ for some (unique) $x \in S$.

The following proposition is evident.

\subsection{Proposition. Every streamline is a partial orbit.}

In the following two key lemmas we investigate the intersection of a partial orbit and a flowbox.

2.3. Lemma. Let $J$ be a partial orbit of a flowbox manifold $X$ and let $g$ : $[0,1] \rightarrow J=g([0,1]) \subset X$ be a topological embedding. Let $V=\phi\left(F_{S}\right)$ be a flowbox in $X$. Then the following holds: if $g(t)=\phi(x, u)$ for some $t \in(0,1), x \in S$, and $u \in(-1,1)$, then there exists $\varepsilon>0$ such that

$$
g((t-\varepsilon, t+\varepsilon)) \subset \phi(\{x\} \times(-1,1)) .
$$

Proof. Let $V$ and $g(t)=\phi(x, u)$ be as above. In view of the definition of a flowbox, $V$ is contained in an open set $U_{\alpha}$ with a product structure; $U_{\alpha}=h_{\alpha}\left(S_{\alpha} \times \Re\right)$. Let $V_{1}=\phi_{1}\left(F_{S_{1}}\right)$ be a flowbox neighborhood of $g(t)$ such that $V_{1} \cap J=\phi_{1}(\{y\} \times[-1,1])$ for some (unique) $y \in S_{1}$. Let $U_{\beta}=h_{\beta}\left(S_{\beta} \times \Re\right)$ be an open set with a product structure such that $V_{1} \subset U_{\beta}$. We write

$$
W=U_{\alpha} \cap U_{\beta} \backslash\left[\phi(S \times\{-1,1\}) \cup \phi_{1}\left(S_{1} \times\{-1,1\}\right)\right] .
$$

In view of the local product structure there is a $U_{\gamma}=h_{\gamma}\left(S_{\gamma} \times \Re\right)$ such that $g(t) \in U_{\gamma} \subset W$. By consistency we have for some $p, q$, and $r$

$$
g(t) \in h_{\gamma}(\{r\} \times \Re) \subset h_{\alpha}(\{p\} \times \Re) \cap h_{\beta}(\{q\} \times \Re) .
$$

By condition (2) in Definition 1.6 we have

$$
g(t) \in \phi(\{x\} \times[-1,1]) \subset h_{\alpha}(\{p\} \times \Re)
$$


and

$$
g(t) \in \phi_{1}(\{y\} \times[-1,1]) \subset h_{\beta}(\{q\} \times \Re) .
$$

As $U_{\gamma} \subset W$ we may conclude

$$
g(t) \in h_{\gamma}(\{r\} \times \Re) \subset \phi(\{x\} \times[-1,1])
$$

and

$$
g(t) \in h_{\gamma}(\{r\} \times \Re) \subset \phi_{1}(\{y\} \times[-1,1])=V_{1} \cap J .
$$

The set $\{r\} \times \Re$ can be written as $\{r\} \times \Re=\bigcup\{\{r\} \times[-n, n] \mid n \in \mathscr{N}\}$. Each interval $\{r\} \times[-n, n]$ is topologically embedded by $h_{\gamma}$ into $J, n \in \mathscr{N}$. It follows that $h_{\gamma}(\{r\} \times \Re)$ is an open interval in the arc $J$. The lemma now easily follows.

2.4. Lemma. Let $\phi: F_{S} \rightarrow V$ be a parameterization of a flowbox $V$ in a flowbox manifold $X$. Suppose that $J$ is a partial orbit of $X$ and that $g:[0,1] \rightarrow J$ is a homeomorphism. Suppose that $J \cap V \neq \varnothing$. Then there is a partition $0 \leq a_{1} \leq b_{1}<a_{2}<b_{2}<\cdots<a_{n} \leq b_{n} \leq 1$ of $[0,1]$ such that

(1) $V \cap J=\left\{g(t) \mid t \in\left[a_{i}, b_{i}\right], i=1, \ldots, n\right\}$;

(2) $g\left(\left[a_{i}, b_{i}\right]\right)$ is a streamline of $V, 2 \leq i \leq n-1$;

(3) if $a_{1}>0$, then $g\left(\left[a_{1}, b_{1}\right]\right)$ is a streamline of $V$; if $a_{1}=0$, then $g\left(\left[a_{1}, b_{1}\right]\right)$ is a possibly degenerate subinterval of a streamline of $V$, such that $g\left(b_{1}\right) \in V \backslash h\left(E_{S}\right)$;

(4) if $b_{n}<1$, then $g\left(\left[a_{n}, b_{n}\right]\right)$ is a streamline of $V$; if $b_{n}=1$, then $g\left(\left[a_{n}, b_{n}\right]\right)$ is a possibly degenerate subinterval of a streamline of $V$, such that $g\left(a_{n}\right) \in V \backslash h\left(E_{S}\right)$.

Proof. Write $C=\{x \in S \mid J \cap \phi(\{x\} \times(-1,1)) \neq \varnothing\}$. Now if $g(t)=\phi(x, u)$ for some $t \in(0,1), x \in C$, and $u \in(-1,1)$, then by Lemma 2.3 we have $g((t-\varepsilon, t+\varepsilon)) \subset \phi(\{x\} \times(-1,1))$. Take the maximal interval $(a, b)$ such that $g((a, b)) \subset \phi(\{x\} \times(-1,1))$. By continuity $g([a, b]) \subset \phi(\{x\} \times[-1,1])$, the inclusion being strict for at most two values of $x$. Write $\left(a_{x}, b_{x}\right)=$ $g^{-1}(\phi(\{x\} \times(-1,1)))$. Observe that $\left\{\left[a_{x}, b_{x}\right] \mid x \in C\right\}$ is a pairwise disjoint collection. The collection is easily seen to be finite, as $\phi(S \times\{-1\})$ and $\phi(S \times\{1\})$ are disjoint closed sets.

Finally we observe that it cannot occur that for some $t \in(0,1), \varepsilon>0$, and $x \in S$ the intersection

$$
g((t-\varepsilon, t+\varepsilon)) \cap \phi(\{x\} \times[-1,1])
$$

is equal to $\{g(t)\}$. This is seen to be true by inspecting the intersection of the partial orbit $\phi(\{x\} \times[-1,1])$ and a suitably chosen flowbox neighborhood of $g(t)$.

The proof of the following proposition now offers no difficulty.

2.5. Proposition. Suppose that $J_{1}$ and $J_{2}$ are partial orbits in a flowbox manifold $X$. If $J_{1} \cap J_{2} \neq \varnothing$, then $J_{1} \cup J_{2}$ is a partial orbit.

By the proposition the following definition is justified. 
2.6. Definition. Let $x$ be a point in a flowbox manifold. The orbit of $x$, denoted by $\Gamma_{x}$, is the union of all partial orbits containing $x$.

The following proposition can be proved in a standard fashion.

2.7. Proposition. Let $X$ be a flowbox manifold. The orbits of $X$ form a partition of $X$.

The structure of orbits is now revealed by the following theorem and its immediate consequences.

2.8. Theorem. Each orbit in a flowbox manifold is in its relative topology a matchbox manifold.

Proof. Let $\Gamma$ be an orbit of $X$. Let $y \in \Gamma$ and $\phi: F_{S} \rightarrow V$ be a parameterization of a flowbox neighborhood of $y$ in $X$. Write $C=\operatorname{pr}_{1}\left(\phi^{-1}(V \cap \Gamma)\right)$ and $F_{C}=C \times[-1,1]$. Because $J \subset \Gamma$ for each partial orbit $J$ with $J \cap \Gamma \neq \varnothing$, it can be seen that $F_{C}=\phi^{-1}(V \cap \Gamma)$. We write $U=\left(\right.$ int $\left._{X} V\right) \cap \Gamma$. It is not difficult to see that all sets $U$ which can be obtained in this way witness the fact that Definition 1.1 is satisfied. As in [AM, Theorem 3.1], it is shown that such a set as $C$ above is countable, whence zero-dimensional.

From the results in $[\mathrm{A} 1, \mathrm{AM}]$ it follows that each orbit in a flowbox manifold is either a topological circle, a topological copy of the reals $\Re$, or a special one-to-one continuous image of the reals, called a $P$-manifold. We recall the following definition from [A1, A2].

2.9. Definition. Let $\Gamma$ be an orbit in a flowbox manifold. If $\Gamma$ is a topological circle, then any covering map $p: \Re \rightarrow \Gamma$ is called a parameterization of $\Gamma$. If $\Gamma$ is not a circle, then any continuous bijection $p: \Re \rightarrow \Gamma$ is called a parameterization of $\Gamma$.

From the results in [A1, A2] it follows that every orbit admits parameterizations.

It turns out that if $p_{1}$ and $p_{2}$ are parameterizations of an orbit $\Gamma$, then the map $p_{1}^{-1} \circ p_{2}: \Re \rightarrow \Re$ is a homeomorphism. If this homeomorphism is increasing, we say that $p_{1}$ and $p_{2}$ have the same direction. Thus there are two directions for each orbit. See [A1, A2] for more details.

2.10. Definitions. Let $X$ be a flowbox manifold. Let $\left\{\Gamma_{\alpha} \mid \alpha \in A\right\}$ be the collection of orbits of $X$. If for each $\alpha \in A$ a parameterization $p_{\alpha}: \Re \rightarrow \Gamma_{\alpha}$ is given, we call the collection $\left\{p_{\alpha} \mid \alpha \in A\right\}$ a parameterization of $X$. If a parameterization has been given, we shall say that a flowbox $V=\phi\left(F_{S}\right)$ is coherently directed if for each $x \in S$ and for any closed interval $J$ in $\Re$ such that

$$
p_{\alpha}(J)=\phi(\{x\} \times[-1,1])
$$

for some $\alpha$, the composition $\mathrm{pr}_{2} \circ \phi^{-1} \circ p_{\alpha}$ is increasing. 
Remark. In the last part of the above definition the $\alpha$ is unique. If $\Gamma_{\alpha}$ is not homeomorphic to a circle $J$ is also unique.

2.11. Definitions. A flowbox manifold $X$ is said to be orientable if there is a parameterization $\left\{p_{\alpha} \mid \alpha \in A\right\}$ of $X$ such that each point of $X$ has a flowbox neighborhood which is coherently directed. In that case the parameterization is called proper.

The following example is no surprise.

2.12. Example. In 1.4 we have seen that any flow without restpoints is a flowbox manifold. If the time (second coordinate) is used as parameter, a proper parameterization is obtained. See $[\mathrm{AM}]$ for more details.

\section{INTERSECTION OF FLOWBOXES}

We investigate the intersection of two flowboxes. First we consider the case where the flowboxes meet in a very special way.

3.1. Proposition. Suppose that $V_{1}$ and $V_{2}$ are flowboxes in $X$. For notational convenience let $V_{1}$ be identified with the standard flowbox by which it is defined: $V_{1}=F_{S}=S \times[-1,1] \subset X$. Let $\phi: F_{T} \rightarrow V_{2}$ be a parameterization of $V_{2}$. Then the following holds true. For each $y \in S$ and $x \in T^{0}$ such that

$$
(\{y\} \times[-1,1]) \cap V_{2}=\phi(\{x\} \times[-1,1]) \in\{y\} \times(-1,1),
$$

there exist an open neighborhood $U$ of $x$ in $T^{0}$ and $W \subset S$ such that

$$
(W \times(-1,1)) \cap V_{2}=\phi(U \times[-1,1]) \cap V_{1} .
$$

Proof. The sets $S \times\{-1,1\}$ and $\phi(\{x\} \times[-1,1])$ are disjoint and $\phi(\{x\} \times[-1,1])$ is compact. Hence the distance between them is positive. It follows that there exists a neighborhood $U^{\prime}$ of $x$ in $T^{0}$ such that

$$
\phi\left(U^{\prime} \times[-1,1]\right) \subset S \times(-1,1) .
$$

Since

$$
(\{y\} \times[-1,1]) \cap V_{2}=\phi(\{x\} \times[-1,1]),
$$

there exists a neighborhood $U \subset U^{\prime}$ of $x$ in $T^{0}$ such that for each $u \in U$ either $\phi(\{u\} \times[-1,1]) \cap V_{1}=\varnothing$ or there exists a unique $k(u) \in S$ such that $\phi(\{u\} \times[-1,1])=(k(u) \times[-1,1]) \cap V_{2}$. Finally we write $W=k(U) \subset S$.

3.2. Remark. Using the notation of the above proposition, it can be shown that $W$ is a neighborhood of $y$ in $S$ and that there exist continuous functions 
$b, t: W \rightarrow[-1,1]$ such that

$$
\phi(U \times[-1,1]) \cap V_{1}=\{(w, s) \mid w \in W \text { and } b(w) \leq s \leq t(w)\} .
$$

See $[A 1, A M]$ for more details.

3.3. Corollary and Definition. Let $V_{1}$ and $V_{2}$ be as described in Proposition 3.1. We define an integer-valued function $n$ on $T^{0}$ as follows. Let $x \in T^{0}$. If there exists $y \in S$ such that $\phi(\{x\} \times[-1,1]) \subset\{y\} \times[-1,1]$, we define $n(x)$ to be the number of points $x^{\prime}$ in $T^{0}$ with $\phi\left(\left\{x^{\prime}\right\} \times[-1,1]\right) \subset\{y\} \times[-1,1]$. Otherwise, $n(x)=0$. Then $n$ is a function of $T^{0}$ to the integers $\mathbf{Z}$ which is upper semicontinuous. The number $n(x)$ is called the index of $x$ with respect to $V_{1}$.

Proof. First we consider a point $x$ with $n(x)=0$. Applying Lemma 2.4 with the partial orbit $\phi(\{x\} \times[-1,1])$ and the flowbox $V_{1}$ we see that there is an $s \in[-1,1]$ such that $\phi(x, s) \notin V_{1}$. By continuity of $\phi$ there is a neighborhood $U$ of $x$ in $T^{0}$ such that $\phi(z, s) \notin V_{1}$ for all $z \in U$, whence $n(z)=0$. Now assume $n(x)>0$. There exists $y \in S$ such that $\phi(\{x\} \times[-1,1]) \subset$ $\{y\} \times[-1,1]$. It is to be noted that $y$ is unique. Now we apply Lemma 2.4 with the orbit $\{y\} \times[-1,1]$ and the flowbox $V_{2}$. We find a partition

$$
-1 \leq a_{1} \leq b_{1}<a_{2}<b_{2}<\cdots<a_{k} \leq b_{k} \leq 1
$$

of $[-1,1]$ such that

$$
V_{2} \cap(\{y\} \times[-1,1])=\bigcup\left\{\{y\} \times\left[a_{i}, b_{i}\right] \mid i=1,2, \ldots, k\right\},
$$

where each $\{y\} \times\left[a_{i}, b_{i}\right]$, with the possible exception of $i=1$ or $i=k$, is a streamline of $V_{2}$. We see that $n(x)=k, k-1$, or $k-2$. By applying the previous proposition with $V_{1}$ to each of the flowboxes

$$
S \times\left[\frac{b_{i}+a_{i+1}}{2}, \frac{b_{i+1}+a_{i+2}}{2}\right], \quad i=1, \ldots, k-2
$$

(and also $S \times\left[-1,\left(b_{1}+a_{2}\right) / 2\right]$ and $S \times\left[\left(b_{k-1}+a_{k}\right) / 2,1\right]$, if necessary), we see that the function $n$ is upper semicontinuous.

\section{Main Theorem}

In this section we are going to show that there can be defined a flow on an orientable matchbox manifold.

4.1. Notation. Throughout this section $X$ is a fixed orientable flowbox manifold. $\left\{\Gamma_{\alpha} \mid \alpha \in A\right\}$ denotes the collection of orbits. For each $\alpha \in A$, $p_{\alpha}: \Re \rightarrow \Gamma_{\alpha}$ is a parameterization of $\Gamma_{\alpha}$ and the parameterization $\left\{p_{\alpha} \mid \alpha \in A\right\}$ is proper.

$\mathscr{V}=\left\{V_{i} \mid i=1,2, \ldots\right\}$ is a fixed flowbox base for $X$. We assume that each $V_{i}=\phi_{i}\left(F_{S_{i}}\right)$ is coherently directed by $\phi_{i}, i=1,2, \ldots$. For $i=1,2, \ldots$ we denote by $\mathscr{C}_{i}$ the collection of all partial orbits of the form

$$
\phi_{i}(\{x\} \times[a, b]), \quad x \in S_{i}^{0},[a, b] \subset(-1,1) .
$$


We write $\mathscr{C}=\bigcup\left\{\mathscr{C}_{i} \mid i=1,2, \ldots\right\}, \mathscr{C}$ is endowed with the Hausdorff metric. It is to be noted that each $\mathscr{C}_{i}$ is an open subset of $\mathscr{C}, i=1,2, \ldots$. If $J \in \mathscr{C}$, then a partition of $J$ is a collection $\left\{J_{1}, \ldots, J_{k}\right\}$ of elements of $\mathscr{C}$ such that

$$
J=J_{1} \cup \cdots \cup J_{k} \quad \text { and } \quad\left|J_{i} \cap J_{i+1}\right|=1, \quad i=1, \ldots, k-1 .
$$

4.2. Lemma and definition. For each $i=1,2, \ldots$ there is a function $\mu_{i}: \mathscr{C} \rightarrow$ $\Re$ with the following properties:

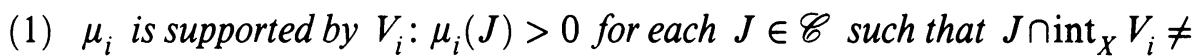
$\varnothing ; \mu_{i}(J)=0$ for each $J \in \mathscr{C}$ such that $J \cap$ int $_{X} V_{i}=\varnothing$.

(2) For each $J \in \mathscr{C}_{1} \cup \cdots \cup \mathscr{C}_{i}$ we have $0 \leq \mu_{i}(J) \leq 1$.

(3) $\mu_{i}$ is additive on $\mathscr{C}$ : if $J_{1}, J_{2}$, and $J_{1} \cup J_{2}$ belong to $\mathscr{C}$ and $\left|J_{1} \cap J_{2}\right|=$ 1 , then $\mu_{i}\left(J_{1} \cup J_{2}\right)=\mu_{i}\left(J_{1}\right)+\mu_{i}\left(J_{2}\right)$.

(4) $\mu_{i}$ is continuous on $\mathscr{C}$.

Proof. We first define $\mu_{1}$. Choose a continuous function $f_{1}: S_{1} \rightarrow[0,1]$ such that $f_{1}(x)>0$ for all $x \in S_{1}^{0}$ and $f_{1}\left(S_{1} \backslash S_{1}^{0}\right)=0$. Define $g_{1}: S_{1} \times[-1,1] \rightarrow$ $[0,1]$ by $g_{1}(x, t)=\frac{1}{2} f_{1}(x)(1-|t|)$.

For any $z \in S_{1}$ and $a, b \in[-1,1]$ with $a \leq b$, we let

$$
\nu_{1}(\{z\} \times[a, b])=\int_{a}^{b} g_{1}(z, t) d t
$$

Now $\mu_{1}$ is defined as follows.

For $J \in \mathscr{C}$ with $J \subset V_{1}$ let $\mu_{1}(J)=\nu_{1}\left(\phi_{1}^{-1}(J)\right)$; for $J \in \mathscr{C}$ with $J \cap$ int $V_{1}=\varnothing$ let $\mu_{1}(J)=0$; for any other $J \in \mathscr{C}$ by Lemma 2.4 there is a partition $\left\{J_{1}, \ldots, J_{k}\right\}$ of $J$ such that for each $\kappa \in\{1, \ldots, k\}$ we have $J_{\kappa} \subset V_{1}$ or $J_{\kappa} \cap$ int $V_{1}=\varnothing$ and we define

$$
\mu_{1}(J)=\sum_{\kappa=1}^{k} \mu_{1}\left(J_{\kappa}\right)
$$

It is easily checked that $\mu_{1}$ satisfies (1) through (4).

We now define $\mu_{i}$ for $i>1$. In order to guarantee that $\mu_{i}$ is also bounded by 1 on $\mathscr{C}_{1} \cup \cdots \cup \mathscr{C}_{i-1}$ we must control the value of the function $g_{i}$ on the previous boxes $V_{1}, \ldots, V_{i-1}$. For this purpose we will define the functions $h_{i j}, 1 \leq j \leq i-1$. First we choose a continuous function $f_{i}: S_{i} \rightarrow[0,1]$ such that $f_{i}(x)>0$ for all $x \in S_{i}^{0}$ and $f_{i}\left(S_{i} \backslash S_{i}^{0}\right)=0$. For each $j, 1 \leq j \leq i-1$, and each $x \in S_{i}^{0}$ we let $n_{i j}(x)$ denote the index of $x$ with respect to $V_{j}$. Further we define

$$
h_{i j}(x)=\frac{1}{n_{i j}(x)+2} \text { and } h_{i}(x)=\min \left\{h_{i j}(x) \mid 1 \leq j<i\right\} .
$$

The function $h_{i}: S_{i}^{0} \rightarrow[0,1]$ is lower semicontinuous and $0<h_{i} \leq \frac{1}{2}$. Then there exists a continuous $\tilde{h}_{i}: S_{i}^{0} \rightarrow[0,1]$ such that $0<\tilde{h}_{i}<h_{i}$. To see that 
this is correct recall that $h_{i}$ is lower semicontinuous if and only if $\{(z, t) \in$ $\left.S_{i}^{0} \times \Re \mid t \geq h_{i}(z)\right\}$ is closed.

Now define $g_{i}: S_{i} \times[-1,1] \rightarrow[0,1]$ by $g_{i}(x, t)=\frac{1}{2} \tilde{h}_{i}(x) f_{i}(x)(1-|t|)$. The functions $\nu_{i}$ and $\mu_{i}$ are now defined in the exact same way as $\nu_{1}$ and $\mu_{1}$. In particular, for any $z \in S_{i}$ and $a, b \in[-1,1]$ with $a \leq b$, we let

$$
\nu_{i}(\{z\} \times[a, b])=\int_{a}^{b} g_{i}(z, t) d t .
$$

For $J \in \mathscr{C}$ with $J \subset V_{i}$ we let

$$
\mu_{i}(J)=\nu_{i}\left(\phi_{i}^{-1}(J)\right) \text {. }
$$

For general $J \in \mathscr{C}$ the definition of $\mu_{i}(J)$ is similar to the definition of $\mu_{1}(J)$ above. The only one statement of (1) through (4) that requires a proof is that $\mu_{i}$ is bounded from above by 1 on $\mathscr{C}_{1} \cup \cdots \cup \mathscr{C}_{i-1}$. Let $J \in \mathscr{C}_{j}, \quad 1 \leq j \leq i-1$. Then $\mu_{i}(J)$ is computed by taking a partition $\left\{J_{1}, \ldots, J_{k}\right\}$ of $J$ such that for each $\kappa \in\{1, \ldots, k\}$ we have $J_{\kappa} \subset V_{i}$ or $J_{\kappa} \cap$ int $V_{i}=\varnothing$. The number of $J_{\kappa}$ with $J_{\kappa} \subset V_{i}$ is bounded from above by $n_{i j}(x)+2$, where $x$ is some element of $S_{i}^{0}$ such that $\phi_{i}(\{x\} \times[-1,1]) \subset J$. For any such $x$ however

$$
\nu_{i}(\{x\} \times[-1,1])=\int_{-1}^{1} g_{i}(x, t) d t \leq \frac{1}{n_{i j}(x)+2},
$$

whence $\mu_{i}(J) \leq\left(n_{i j}(x)+2\right) /\left(n_{i j}(x)+2\right)=1$.

We are now in a position to prove the Main Theorem (0.5) which has been stated in the Introduction.

4.3. Proof of the Main Theorem. We use the notation of the lemma.

The function $\mu: \mathscr{C} \rightarrow \Re$ is defined by

$$
\mu(J)=\sum_{k=1}^{\infty} \frac{\mu_{k}(J)}{2^{k}} .
$$

It is to be noted that if $J \in \mathscr{C}_{i}$, then $\mu_{k}(J) \leq 1$ for all $k \geq i$ by condition (2) of Lemma 4.2, whence $\sum_{k=i}^{\infty} \mu_{k}(J) / 2^{k} \leq 2^{-i+1}$. It follows that the series at the right-hand side is uniformly convergent on each $\mathscr{C}_{i}, i=1,2, \ldots$. It is now clear that $\mu$ is continuous and additive on $\mathscr{C}$.

In what follows it might be useful to think of $\mu$ as a time span function, that is, $\mu(J)$ measures the time it takes to travel from one endpoint of $J$ to the other.

For each $x \in X$ we define $\alpha_{x}$ and $\omega_{x}$ as follows:

$$
\begin{gathered}
\alpha_{x}=\inf \left\{-\mu\left(\phi_{i}(\{z\} \times[-1, t])\right) \mid x=\phi_{i}(z, t) \in V_{i}\right\}, \\
\omega_{x}=\sup \left\{\mu\left(\phi_{i}(\{z\} \times[t, 1])\right) \mid x=\phi_{i}(z, t) \in V_{i}\right\} .
\end{gathered}
$$

For each $x \in X$ and $s \in\left(\alpha_{x}, \omega_{x}\right)$ we define $\pi(x, s)$ as follows. For $0 \leq s \leq$ $\omega_{x}$ choose $i$ and $(z, t)$ such that $x=\phi_{i}(z, t)$ and $\mu\left(\phi_{i}(\{z\} \times[t, 1])\right)>s$. 
Then select the unique $y=\phi_{i}(z, u)$ such that $\mu\left(\phi_{i}(\{z\} \times[t, u])\right)=s$ and $t \leq u \leq 1$. We let $y=\pi(x, s)$. For $\alpha_{x}<s \leq 0$ the value of $\pi(x, s)$ is defined in a similar way.

Here we make the observation that it is only at this point that the orientability of the flowbox manifold is required.

Finally we write

$$
D=\bigcup\left\{\{x\} \times\left(\alpha_{x}, \omega_{x}\right) \mid x \in X\right\} \subset X \times \Re .
$$

The following facts are easily established.

(U0) $D$ is a neighborhood of $X \times\{0\}$ of the form above.

(U1) Identity axiom: $\pi(x, 0)=x$ for all $x \in X$.

(U2) Homomorphism axiom: if $(x, t) \in D,(x, t+s) \in D$ and $(\pi(x, t), s)$ $\in D$, then $\pi(\pi(x, t), s)=\pi(x, t+s)$.

(U3) Continuity axiom: $\pi$ is continuous.

Properties (U0) through (U3) of $\pi: D \rightarrow X$ are precisely the defining properties of a germ of a local flow [U, H2]. There is a standard way of extending the domain $D$. For $t>0$ the point $(x, t) \in X \times \Re$ is called admissible if there exists a partition $0=t_{0}<t_{1}<\cdots<t_{k}=t$ of $[0,1]$ such that $t_{\kappa+1}-t_{\kappa}<\omega_{\pi\left(x, t_{\kappa}\right)}$ for each $\kappa=0, \ldots, k-1$. Then for $\kappa=0, \ldots, k-1$ one defines inductively

$$
\pi\left(x, t_{\kappa+1}\right)=\pi\left(\pi\left(x, t_{\kappa}\right), t_{\kappa+1}-t_{\kappa}\right) .
$$

A similar definition applies when $t<0$. See [H2] for details. In this way one arrives at a local flow. The reader is referred to [H1, U] for more information about local flows.

The proof of the theorem is completed by invoking a result of Carlson [Ca] which states that for a local flow there exists a reparameterization which turns it into an equivalent global flow.

4.4. Remark. By a simple compactness argument it can be shown that the orbits of the flowbox manifold, as defined in Definition 2.6, coincide with the orbits of the flow. From this observation combined with results of Ura [U] it may be deduced that the flow which has been constructed in the above proof is unique up to a reparameterization.

\section{REGULAR FAMILIES OF CURVES}

In Whitney's paper [W] a family of curves in a space $X$ was defined as a partition of $X$ into arcs, with or without endpoints, and circles. In the second part of the paper arcs with endpoints are excluded. We shall extend Whitney's major results in such a way that more general curves are admitted.

5.1. Definition. A curve is either a circle or a separable metric space which is a one-to-one continuous image of the real line $\Re$. A family of curves in a separable metrizable space $X$ is a partition $\left\{C_{\alpha} \mid \alpha \in A\right\}$ of the space into curves. 
5.2. Remark. In what follows it is convenient to denote a family of curves in $X$ by $\left\{C_{x} \mid x \in X\right\}$, where $x \in C_{x}$ for each $x \in X$. So every element of the partition is labeled by each of its elements.

5.3. Definition. A family $\left\{C_{x} \mid x \in X\right\}$ of curves in $X$ is called regular if for each $\varepsilon>0$, each $x \in X$, and each arc $J$ with $x \in J \subset C_{x}$ there exists a $\delta>0$ such that for every $y \in B_{\delta}(x)$ there is an $\varepsilon$-embedding $h: J \rightarrow C_{y}$ (i.e., $d(s, h(s))<\varepsilon$ for every $s \in J)$ such that $h(x)=y$.

The following is a preparation for the proof of Theorem 5.7 which states the equivalence of the notions of flowbox manifold and regular family of curves. The definition, lemma, and theorem below are an almost verbatim generalization of the results in $\S 3$ of [AHO]. In fact the proofs in [AHO] closely follow the argument designed by Whitney in the original situation [W]. Proofs will be omitted.

5.4. Definition. Suppose that $\left\{C_{x} \mid x \in X\right\}$ is a regular family of curves in $X$. Let $x \in X$. A closed set $S$ is a local section at $x$ provided that $x \in S$ and that there exists a neighborhood $U$ of $x$ such that for each $y \in \operatorname{cl}_{X} U$ each component of $C_{y} \cap \mathrm{cl}_{X} U$ intersects $S$ in exactly one point.

5.5. Lemma. Suppose that $\left\{C_{x} \mid x \in X\right\}$ is a regular family of curves in $X$. Then $X$ admits a local section at every point $x \in X$.

5.6. Theorem. Suppose that $\left\{C_{x} \mid x \in X\right\}$ is a regular family of curves in $X$. Then for each $x \in X$ there exist a neighborhood $U$ of $x$, a section $S$ at $x$, and a topological embedding $\psi: S \times[-1,1] \rightarrow \mathrm{cl}_{X} U$ such that

(i) $\psi(S \times\{0\})=S$ and $\psi(x, 0)=x$,

(ii) $\psi(s, t) \in C_{s}$ for each $s \in S$ and $t \in[-1,1]$.

5.7. Theorem. A separable metrizable space $X$ is a flowbox manifold if and only if there is a regular family of curves in $X$.

Proof. The "if" part immediately follows from the preceding theorem. It is to be noted that the consistency condition follows from the fact that we start out with a family of curves by which the local products are lined up.

To prove the "only if" part we show that if $X$ is a flowbox manifold, then the family $\left\{\Gamma_{x} \mid x \in X\right\}$ of all orbits (Definition 2.6) is a regular family. If the flowbox manifold $X$ is orientable, then by the Main Theorem (0.5) there is a flow $\pi: X \times \Re \rightarrow X$ such that each streamline of $X$ is contained in some orbit of the flow. It easily follows that every $\Gamma_{x}$ coincides with some orbit in the flow. The condition expressed in the definition of a regular family is a wellknown property in the theory of flows. Sometimes it is called the continuity of the initial conditions [H1, NS]. If the flowbox manifold is not orientable we have to fall back on the method of pasting together of flowboxes. This method has been described in a very detailed way in [A1] for a $P$-manifold and can be adapted for the present situation with only minor modifications. The crucial lemma leading up to the Pasting Theorem is Proposition 3.1 above. 
The method of pasting flowboxes together can also be employed to prove the following result which already has been observed by Whitney [W] and Chewning [Ch].

5.8. Corollary. Suppose that $\left\{C_{x} \mid x \in X\right\}$ is a regular family of curves. Suppose that $x$ and $y$ belong to the same curve (i.e., $C_{x}=C_{y}$ ). Let $S_{x}$ and $S_{y}$ denote a local section of $x$ and $y$ respectively. Then there are neighborhoods $W_{x}$ and $W_{y}$ of $x$ and $y$ respectively such that $W_{x} \cap S_{x}$ is homeomorphic to $W_{y} \cap S_{y}$.

The following is a generalization of another theorem by Whitney [W].

5.9. Theorem. Suppose that $C$ is a closed subset of a space $X$ and that $X \backslash C$ is an orientable flowbox manifold. Then there exists a flow $\tilde{\rho}: X \times \Re \rightarrow X$ such that $\tilde{\rho} \mid(X \backslash C) \times \Re$ has no fixed points and each $x \in C$ is a fixed point of the flow $\tilde{\rho}$.

Proof. By Theorems 5.7 and 0.5 there exists a flow $\pi:(X \backslash C) \times \Re \rightarrow X \backslash C$ such that the curve $C_{x}$ coincides with the orbit $\Gamma_{x}$ of $\pi$ through $x, x \in X \backslash C$. We shall now define a parameter transformation which slows down the motion near $C$. The techniques we are going to use can be found in [NS, Ca]. We first define a continuous function $g: X \rightarrow \Re$ such that $g(x)=0$ for $x \in C$ and $0<g(x) \leq 1$ for $x \in X \backslash C$.

For $x \in X \backslash C$ we write $G(x)=1 / g(x)$. Now we define the parameter transformation $\tau:(X \backslash C) \times \Re \rightarrow \Re$ by

$$
\tau(x, t)=\int_{0}^{t} G(\pi(x, u)) d u .
$$

By direct computation it can be verified that the function $\tau$ has the following properties:

(i) $\tau$ is continuous.

(ii) For each $x \in X \backslash C$ the function $\tau_{x}: \Re \rightarrow \Re$, defined by $\tau_{x}(t)=$ $\tau(x, t)$, is an increasing topological map of $\Re$ onto $\Re$ and $\tau_{x}(0)=0$.

(iii) For all $t, s \in \Re$ one has

$$
\tau(x, s+t)=\tau(x, t)+\tau(\pi(x, t), s) .
$$

We define a new flow $\rho:(X \backslash C) \times \Re \rightarrow X \backslash C$ by $\rho(x, u)=\pi\left(x, \tau_{x}^{-1}(u)\right)$. It is not hard to show that $\rho$ has the group property. To see that $\rho$ is continuous we define $\phi:(X \backslash C) \times \Re \rightarrow(X \times C) \times \Re$ by $\Phi(x, t)=(x, \tau(x, t))$.

We consider the commutative diagram

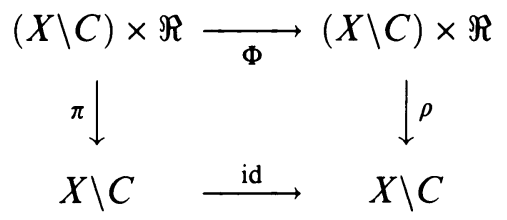

Note that $\tau=\operatorname{pr}_{2} \circ \Phi$. Then $\Phi$ is topological by [Ca, Lemma 1]. The continuity of $\rho$ follows, as $\rho=\pi \circ \Phi^{-1}$. 
Finally we define $\tilde{\rho}: X \times \Re \rightarrow X$ by

$$
\tilde{\rho}(x, t)= \begin{cases}x & \text { if } x \in C, \\ \rho(x, t) & \text { if } x \notin C .\end{cases}
$$

The only thing that requires a proof is that $\left(x_{i}, t_{i}\right) \rightarrow\left(x_{0}, t_{0}\right), x_{i} \notin C$, $i=1,2, \ldots$, and $x_{0} \in C$ implies that $\tilde{\rho}\left(x_{i}, t_{i}\right) \rightarrow \tilde{\rho}\left(x_{0}, t_{0}\right)=x_{0}$. For each $i$ we write $s_{i}=\tau_{x_{i}}^{-1}\left(t_{i}\right)$. Then $\tilde{\rho}\left(x_{i}, t_{i}\right)=\pi\left(x_{i}, s_{i}\right)$ for each $i$. For $i \rightarrow \infty$ we have $t_{i} \rightarrow t_{0}$ and $G\left(x_{i}\right) \rightarrow \infty$. As $t_{i}=\tau\left(x_{i}, s_{i}\right)$, it follows that $s_{i} \rightarrow 0$. Consequently,

$$
\lim _{i \rightarrow \infty} \tilde{\rho}\left(x_{i}, t_{i}\right)=\lim _{i \rightarrow \infty} \pi\left(x_{i}, s_{i}\right)=\pi\left(x_{0}, 0\right)=x_{0} .
$$

This completes the proof.

In Example 1.2 we discussed the matchbox manifolds. As is stated below the flow on a matchbox manifold can be given a very special form. For details the reader is referred to $[\mathrm{AM}]$.

5.10. Theorem. If $X$ is a one-dimensional orientable flowbox manifold, then the flow on $X$, whose existence has been established in Theorem 0.5 , is a suspension $\sum(S, h)$ of some autohomeomorphism of a closed subset $S$ of $X$.

The dimensional restriction in the preceding theorem is necessary as the following example shows.

5.11. Example. This is an example of a two-dimensional flowbox manifold $X$ that is not a suspension. It is a well-known example leading up to the Reeb foliation [CN]. $X$ is the strip $\left\{(x, y) \mid-\frac{\pi}{2} \leq y \leq \frac{\pi}{2}\right\}$ in the plane $\Re^{2}$. The elements of the partition are the lines $y= \pm \frac{\pi}{2}$ and the curves $x+c=1 / \cos (y)$, $c \in \Re$.

The family of these curves is regular and the flowbox manifold $X$ (Theorem 5.7) is orientable. In [NS, p. 30], there can be found a description of the flow, which exists by Theorem 0.5 , by means of differential equations.

It can be shown that the local sections of $X$ must be connected and locally homeomorphic to an arc [H1, Chapter VI]. From this it can be deduced that $X$ cannot be a suspension.

\section{REFERENCES}

[A1] J. M. Aarts, The structure of orbits in dynamical systems, Fund. Math. 129 (1988), 39-58.

[A2] - Orientations of orbits in flows, Papers on General Topology and Related Category Theory and Topological Algebra, no. 552, Ann. New York Acad. Sci., 1989, 1-7.

[AM] J. M. Aarts and M. Martens, Flows on one-dimensional spaces, Fund. Math. 131 (1988), 53-67.

[AHO] J. M. Aarts, C. L. Hagopian and L. G. Oversteegen, The orientability of matchbox manifolds, Pacific J. Math (to appear).

[Ca] D. H. Carlson, A generalization of Vinograd's theorem for dynamical systems, J. Differential Equations 11 (1972), 193-201. 
[Ch] W. C. Chewning, A dynamical system on 4-dimensional Euclidean space neither isomorphic nor equivalent to a differential system, Bull. Amer. Math. Soc. 80 (1974), 150-153.

[CN] C. Camacho and A. L. Neto, Geometric theory of foliations, Birkhäuser, Basel, 1985.

[G] A. Gutek, On compact spaces which are locally Cantor bundles, Fund. Math. 108 (1980), 27-31.

[H1] O. Hajek, Dynamical systems in the plane, Academic Press, London, 1968.

[H2] Local characterization of local semi-dynamical systems, Math. Systems Theory 2 (1968), 17-25.

[MR] M. W. Mislove and J. T. Rogers, Jr., Local product structures on homogeneous continua, Topology Appl. 31 (1989), 259-267.

[N] S. B. Nadler, Jr., Hyperspaces of sets, Dekker, New York, 1978.

[NS] V. V. Nemytskii and V. V. Stepanov, Qualitative theory of differential equations, Princeton Univ. Press, Princeton, N. J., 1960.

[U] T. Ura, Isomorphisms and local characterization of local dynamical systems, Funkcial. Ekvac. 12 (1969), 99-122.

[W] H. Whitney, Regular families of curves, Ann. of Math. 34 (1933), 244-270.

Technische Universiteit Delft, Faculteit der Wiskunde en Informatica, Postbus 356, 2600 A J Delft, The Netherlands

Department of Mathematics, University of Alabama, Birmingham, Alabama 35294 